\title{
Research on hospital operation scheduling based on chance constraints under uncertain conditions
}

\author{
Weining $\mathrm{Li}^{1}$, Fuyu Wang ${ }^{1,2}$ \\ ${ }^{1}$ School of management science and engineering, Anhui University of Technology, Ma'anshan, \\ Anhui, China \\ ${ }^{2}$ School of management, University of Shanghai for Science and Technology, Shanghai, \\ China \\ 1448522819@qq.com
}

Corresponding author Weining $\mathrm{Li}$

Key words: fuzzy uncertainty, chance constrained, surgical scheduling, hybrid intelligent algorithm

\begin{abstract}
The uncertain factors in hospital operation scheduling process, selection of optimization problems in the risk level operation, in order to maximize the benefits of a hospital for elective surgery, in the fuzzy uncertain environment, considering the factors of time and resource constraints and risk confidence level, a fuzzy dependent chance programming model through the hybrid intelligent algorithm design to find the optimal solution of the model, finally this paper will illustrate the operability of the model, to prove the validity of the algorithm.
\end{abstract}

\section{不确定条件下基于机会约束的医院手术调度研究}

\author{
李卫宁 ${ }^{1}$ 王付宇 ${ }^{1,2}$ \\ 1 安徽工业大学 管理科学与工程学院, 马鞍山, 安徽, 中国 \\ 2 上海理工大学 管理学院, 上海, 中国 \\ 1448522819@qq.com \\ 通讯作者 李卫宁
}

关键词：模糊不确定；机会约束；手术调度；混合智能算法

摘要：医院手术调度过程中不确定因素较多，在风险置信水平下手术选择优化问题，为了最 大化医院择期手术收益，在模糊不确定的环境下，考虑了时间、资源限制和风险置信水平等 因素, 建立了模糊相关机会规划模型, 通过设计的混合智能算法求出模型的最优解, 最后本 文将举例来说明模型的可操作性, 证明算法的有效性。

\section{1. 引言}

医疗卫生市场竞争日益激烈，医院必须通过合理调度增加收益，提高病人满意度才能在 市场中占有一席之地。手术室作为医院最大的成本和收入中心, 是影响整个医院的绩效的重 要方面 ${ }^{[1]}$ 。目前国内外相关研究人员对手术调度采用最多的方法还是通过对历史经验数据的 总结, 从而分析出最合理的调度方案, 使患者的手术顺序得到有效的排列 ${ }^{[2-4]}$ 。然而很多的多 医院手术调度中很少考虑在置信水平下的收益状况。本文在此基础上研究时间和费用均为模 糊的情况下, 在一定的可信度 (置信水平) 下进行手术调度, 并利用混合遗传算法解决此模 型。 


\section{2. 模糊相关理论}

定义 $1^{[5]}$ : 设有一论域为 $\mathrm{U}$, 其一个子集为 $\tilde{\mathrm{A}}$, 对于其中的任何某一元素 $x \in U$, 函数 $\mu \tilde{\mathrm{A}}$ : $\mathrm{U} \rightarrow[0,1]$, 随之确定一个值 $\mu_{\tilde{A}} \in[0,1]$ 与其相对应。 $\mu_{\tilde{A}}$ 在 $\mathrm{x}$ 处的值表示的是元素 $\mathrm{x}$ 属于 $\tilde{\mathrm{A}}$ 的 隶属程度, 那么我们就称 $\tilde{\mathrm{A}}$ 为模糊子集, $\mu \tilde{\mathrm{A}}$ 就表示 $\tilde{\mathrm{A}}$ 的隶属函数。

定义 $2^{[6]}$ : 如果模糊数 $\tilde{\mathrm{A}}=(\mathrm{a} 1, \mathrm{a} 2, \alpha, \beta)$ 的隶属函数满足

$$
\mu_{\mathrm{i}}(x)=\left\{\begin{array}{l}
\left(a_{1}-x\right) / \alpha, a_{1}-\alpha \leq x \leq a_{1} \\
1, \quad a_{1} \leq x \leq a_{2} \\
\left(x-a_{2}\right) / \beta, a_{2} \leq x \leq a_{2}+\beta \\
0, \quad \text { 其他 }
\end{array}\right.
$$

则称 $\tilde{\mathrm{A}}$ 是一个梯形模糊数, 公式中 $\mathrm{a} 1$ 和 $\mathrm{a} 2$ 分别表示梯形模糊数的左模态值和右模态值; $\alpha$ 和 $\beta$ 分别是其对应的左延展值和右延展值。梯形模糊隶属函数的参数关系如图 1 所示, 梯形 模糊数 $\tilde{\mathrm{A}}$ 中 $\mathrm{x}$ 的可能性程度在图中表示 $\mu \tilde{\mathrm{A}}(\mathrm{x}) \in[0,1]$ 。这个主要的不确定值的范围在 $[\mathrm{a} 1, \mathrm{a} 2]$

(核心范围)，核心外的最有可能的范围延展值为 $[\mathrm{a} 1-\alpha, \mathrm{a} 2-\beta]$ （支持范围）。其梯形模糊数的 范围值就在核心值内部和外延值内部产生。

\section{3. 模型的建立}

在每个候选项目的成本和收益都是模糊的情况下，建立模糊机会约束规划。

$\widetilde{\mathbf{t}}_{\mathbf{i}}$ 表示完成第 $\mathrm{i}$ 台手术的模糊时间

$n$ 表示候选手术的数目;

$\tilde{\mathrm{c}}_{\mathrm{i}}$ 表示第 $\mathrm{i}$ 台手术的模糊费用;

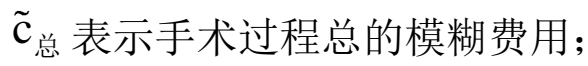

$x_{\mathrm{i}}$ 为 $0-1$ 变量, 当手术 $\mathrm{i}$ 被选中时, 其值为 1 ,; 反之当未被选中时其值为 0 。 建立如下模型

$$
\operatorname{Max}\left(\sum_{i=1}^{n} \tilde{\mathrm{c}}_{\mathrm{i}} x_{i}\right)
$$

$$
\begin{aligned}
& \text { S.t. } \quad \operatorname{Cr}\left\{\sum_{i=1}^{n} \tilde{t}_{i} x_{i} \leq T\right\} \geq 0.9 \\
& \operatorname{Cr}\left\{\sum_{i=1}^{n} c_{i} x_{i} \leq \tilde{c}_{\text {总 }}\right\} \geq 0.8 \\
& \sum_{i=\mathbf{1}}^{n} \mathbf{x}_{\mathbf{i}} \leq Z \\
& x_{i} \in\{0,1\}, \quad \forall i
\end{aligned}
$$

目标函数表示最大化手术收益, 约束条件 (1) 是最大完工时间不能超过 4 最大时间 $\mathrm{T}=240$ 分钟的可信度大于等于 0.9 ; 约束条件 (2) 是指各手术成本费用不得超过手术总费用的可信 度大于等于 0.8 ; 约束条件 (3) 表示一天择期手术数目不能超过 $\mathrm{Z}=2$ 台; 约束条件 (4) 为 0-1 变量, 表示当手术 $\mathrm{i}$ 被选择时, 那么它的值就为 1 , 否则未被选中时其值就为 0 。为解决 上述模糊机会约束规划模型，我们采用混合智能算法。

第一步: 首先利用模糊模拟解决不确定函数的输入输出数据

第二步: 根据步骤 1 得出的数据训练神经网络用于逼近不确定函数

第三步：产生初始染色体，利用第二步训练后的神经元网络来检验其可行性

第四步: 通过遗传算法的交叉变异来更新染色体, 并在此用第二步训练后的神经元网络 
来检验

第五步：通过神经元网络计算所以染色体的目标值

第六步：由第五步的目标值计算染色体适应度

第七步：利用旋转奢轮选择染色体

第八步：重复第四步到第七步至循环次数

第九步: 输出最优染色体, 即为最优解

\section{4. 案列分析}

为了解释上述模型的操作可信性, 我们利用一个案列来解释。假设某医院有 3 台择期手 术, 现对其进行选择。梯形模糊变量手术 1 时间 (44.3,45.4,3,4) 其费用为 $(50,60,2,3)$; 手 术 2 时间 ( 55.5,60,3,3.5) 其费用为 (45,50,2,3); 手术 3 时间为 (50,55,2.5,3) 其费用为 $(50,55,2,3$ )。 医院收入总费用不能超 8 万，假设收益置信水平为 0.9 （即 $\alpha=0.9 ）$ ；成本置信水平为 0.8 (即 $\beta=0.8$ )。为了得出最大收益值, 我们通过训练一个神经元网络来逼近不确定函数 $U$, 其包含 3 个输入神经元, 6 个隐层神经元, 2 个输出神经元。通过 MATLAB 运行混合智能算法（种 群规模设为 50 , 交叉概率设为 0.3 , 变异概率设为 0.2 ; 通过 1000 次的模拟循环, 500 个训练 样本，500 次的遗传迭代）得到最优解为：57.7148（单位：千元）。

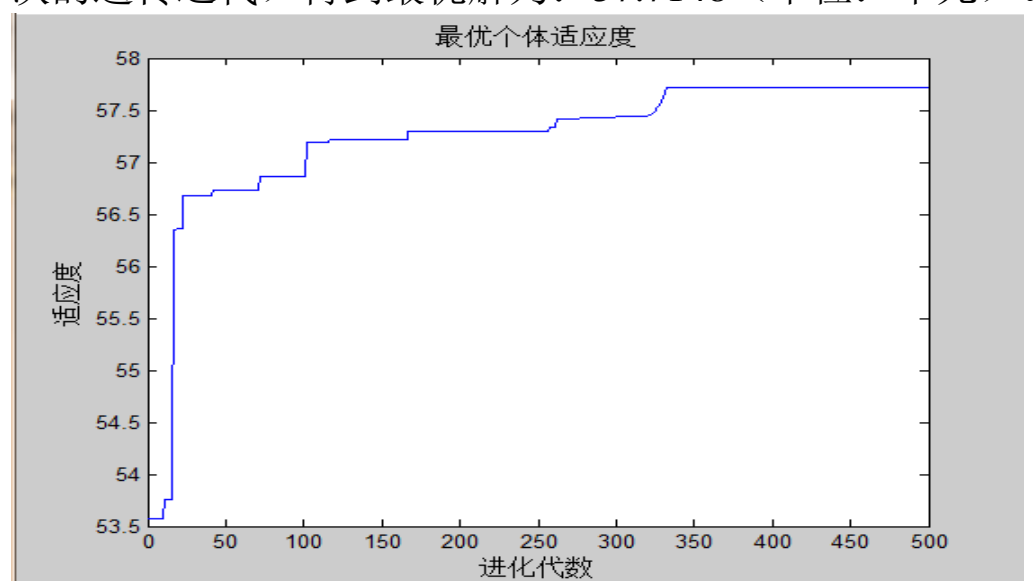

\section{5. 结束语}

本文在时间和费用模糊的情况下考虑了时间和费用的置信水平，实用性较高，但是并未 加入其它不确定因素, 比如风险, 既然是手术那么规避风险也是即为重要的。还有本文的最 大总收入是一个确定的值, 假设将其设为模糊数（因为医院也不能百分百确定总收入正好是 个确定的值）。所以后续可以从这几个方面入手，让模型更加贴近实际。

\section{References}

[1] Xue Bai, Rowley, Chenxi Yang, et al. Study on the application of production scheduling theory in operation room scheduling optimization [J]. West China Medical University, 2015 (10): 1990-1995.

[2] B. Cardoen, E. Demeulemeester, J. Belien. Operating Room Planning and Scheduling: A Literature Review[J]. European Journal of Operational Research, 2010,201(3):921-932.

[3] Dexter F, Macario A, Traub RD. An Operating Room Scheduling Strategy to Maximize the Use of Operating Room Block Time: Computer Simulation of Patient Scheduling and Survey of Patients' Preferences for Surgical Waiting Time[J]. Anesthesia \& Analgesia, 1999,89(17):7-20.

[4] Zakaria, Y. Abdelrasol, Nermine Harraz, Amr Eltawil. A Proposed Solution Framework for the 
Operating Room Scheduling Problems[A]. Proceedings of the World Congress on Engineering and Computer Science 2013[C].2013.

[5] Zadeh L . Fuzzy Sets [J]. Inform and Control, 1965, 8: 338-353.

[6] Klir GJ, Yuan B. Fuzzy sets and fuzzy logic [J]. theory and applications, 1995, 10(8): 245-254. 